\title{
Implementation of analytical models of the anisotropic combined dry friction in problems of pneumatics' dynamics
}

\author{
Alexey Kireenkov ${ }^{1, *}$, Sergey Zhavoronok ${ }^{2, * *}$ \\ ${ }^{1}$ Moscow Institute of Physics and Technology (State University), 141701 Dolgoprudny, Russia \\ Institute for Problems in Mechanics of the Russian Academy of Science, 119526 Moscow Russia \\ ${ }^{2}$ EDP Sciences, Production Department, 91944 Les Ulis Cedex A, France
}

\begin{abstract}
An implementation of the theory of multi-component dry friction in some engineering problems of pneumatics' dynamics is proposed. The main attention is devoted to the construction of analytical models of the combined dry friction accounting for the anisotropy of the dry friction coefficients and the real distribution of normal and tangent contact stresses. These models are applied for more detailed investigation of unsteady rolling regimes of pneumatics which are characterized by the non-vanishing sliding and spin.
\end{abstract}

\section{Introduction}

The phenomenon of the shimmy of wheels of aircraft landing gears and automotive vehicles is well-known but not completely studied. Indeed, the traditionally used model of a shimmy is proposed by M. V. Keldysh [1]. Such an approach results a very simple model with reduced number of degrees of freedom, does not require complex numerical methods and still remains useful in the engineering practice [2]. In the same time the instable motion of various wheels is observed at the stages of non-steady rolling with significant longitudinal sliding in the contact spot; moreover, the spinning can appear in case of the disturbed motion [3]. It is clear that the Keldysh assumptions are inconsistent with such a regime, and the dry friction effects on the motion stability cannot be studied using the traditional shimmy theory [2]. On the other hand, the combined kinematics of relative motion in the contact area requires the qualitative improvement of dry friction theories as it was shown in [4] and [5]; the classical Coulomb dry friction law is unable to describe a wide range of phenomena due to the coupling of sliding and spinning.

The rigid wheel shimmy is one of such phenomena; it is induced only by the coupling of the dry friction forces and torque $[6,7]$. This effect was found using the new dry friction model so-called "poly-component" or "multi-component" [8-11].

The quasi-rigid wheel theory $[9,10]$ was applied to investigate the shimmy of landing gears [3, 12-14]. It seems to be a good first approximation, nevertheless this simplest approximate model has almost nothing to do with the real objects of the engineering

\footnotetext{
* Corresponding author: kireenk@ipmnet.ru
} 
analysis. Thus, the shimmy models $[3,6,7,12-14]$ require some significant improvements as well as the combined dry friction theory [8-11].

Different improvements of the dry friction theory were presented in articles of many authors among which are works [15-22]. Here the basics of the improved theory of coupled dry friction are presented, the dry friction anisotropy is introduced, and the formulae for the resultant vector and couple of the dry friction are constructed. Such a model seems to be a good second approximation for a wheel under combined rolling, sliding, and spin and can be applied to study the shimmy initiation conditions.

\section{Model of the combined rolling, sliding, and spin of the rigid body based on the coupled dry friction theory}

\subsection{On the anisotropic dry friction}

In case of the frictional anisotropy induced by the structure of interacting bodies and/or texture of the contacted surface the Amonton-Coulomb dry friction law can be written in the following formulation [18-21]:

$$
\mathbf{F}=-|N| \frac{\mathbf{f} \cdot \mathbf{v}}{|\mathbf{v}|} \quad(\mathbf{v} \neq 0) .
$$

Here the second rank tensor $\mathbf{f}=f_{\alpha \beta} \mathbf{e}^{\alpha} \mathbf{e}^{\beta}$ is the dry friction coefficient; $\mathbf{e}^{\alpha}, \alpha=1,2$ are base vectors of some frame $O x^{1} x^{2}$ on the plane of interaction of the contacting bodies, $\mathbf{v}=v^{\alpha} \mathbf{e}_{\alpha}$ is the vector of the relative sliding velocity, and the symbol « $\bullet »$ denotes the scalar product. In general $f_{\alpha \beta} \neq f_{\beta \alpha}$ (e. g. see $[18,22]$ ).

Let us consider the dry friction tensor $\mathbf{f}$ be positively defined:

$$
\mathbf{v}_{1}^{\mathrm{T}} \cdot \mathbf{f}_{S} \cdot \mathbf{v}_{1}>0 \quad \forall \mathbf{v}_{1}(\mathbf{q}): \mathbf{q} \in \Omega \subset R^{n}, \quad \mathbf{f}_{S}=\frac{1}{2}\left(\mathbf{f}+\mathbf{f}^{\mathrm{T}}\right),
$$

where $\Omega$ is the configuration space of the considered mechanical system, and $\mathbf{q}$ is the vector of generalized coordinates. Thus, the linear mapping of the set of unit vectors $\mathbf{v}_{1}$ into the set of dry friction force vectors $\mathbf{F}$ (1) is defined by the linear operator $\mathbf{f}$. As usually, the unit vector $\mathbf{v}_{1}=\mathbf{v} /|\mathbf{v}|$ is the relative sliding director.

The general cohesion condition can be represented in the formulation [20]:

$$
I_{2}(\mathbf{f}) \neq 0: \quad I_{2}^{-2}(\mathbf{f})\left\{I_{1}^{2}(\mathbf{f})|\mathbf{F}|^{2}+\left[I_{1}(\mathbf{f}) \mathbf{f}_{S}+\mathbf{f}^{\mathrm{T}} \cdot \mathbf{f}\right]: \mathbf{F} \otimes \mathbf{F}\right\}=|N|^{2},
$$

where $I_{1}(\mathbf{f})$ and $I_{2}(\mathbf{f})$ are invariants of dry friction tensor $\mathbf{f}$, and the symbol «®» denotes the tensor product.

\subsection{The local model of the anisotropic dry friction}

In general, the plane-parallel relative motion, i. e. the simultaneous sliding and spinning, of the rigid bodies with the finite contact spot $S$ requires the qualitative improvement of the Amonton-Coulomb dry friction law [5-11]. The aim of this theory consists in the differential formulation of the Coulomb law as a local model of the friction interaction in each point of the contact area $S$ : 


$$
\forall M \in S \rightarrow \boldsymbol{\tau}=-\left|\sigma_{v}\right| \frac{\mathbf{f} \cdot \mathbf{v}_{\Sigma}}{\left|\mathbf{v}_{\Sigma}\right|},\left(\mathbf{v}_{\Sigma} \neq 0\right), \mathbf{v}_{\Sigma}=\mathbf{v}_{0}-R \boldsymbol{\omega}_{\tau} \times \mathbf{e}_{3}+\boldsymbol{\omega}_{v} \times \mathbf{r}_{\tau}
$$

Here $\mathbf{v}_{\Sigma}$ denotes the summary velocity of the relative slip in the arbitrary point $M \in S$, $\mathbf{v}_{0}(\mathbf{q})$ is the longitudinal absolute velocity, $\boldsymbol{\omega}_{\tau}(\mathbf{q})$ is the angular velocity of rolling, $\boldsymbol{\omega}_{v}(\mathbf{q})$ is the angular velocity of spinning, $R(M)$ is the curvature radius of the rolling body calculated in the point $M, \mathbf{r}_{\tau}(M)$ is the vector radius of the point $M \in S$ in the plane of contact, $\mathbf{e}_{3}$ denotes the normal unit vector of the contact plane, $\boldsymbol{\tau}$ is the frictional tangential stress in the contact area $S$, and $\sigma_{v}$ denotes the normal contact pressure. Thus, the cohesion condition can be formulated locally in the point $M \in S$ as follows:

$$
I_{2}^{-2}(\mathbf{f})\left\{I_{1}^{2}(\mathbf{f})|\boldsymbol{\tau}|^{2}+\left[I_{1}(\mathbf{f}) \mathbf{f}_{S}+\mathbf{f}^{\mathrm{T}} \cdot \mathbf{f}\right]: \boldsymbol{\tau} \otimes \boldsymbol{\tau}\right\}=\left|\sigma_{v}\right|^{2}
$$

Let us consider the combined kinematics, i. e. the simultaneous slip, spin, and rolling. Therefore, taking into account the dry friction anisotropy, we obtain the following formula for the tangential stress:

$$
\boldsymbol{\tau}_{1}=-\left|\sigma_{v}\right|\left|\mathbf{v}_{0}+\boldsymbol{\omega}_{v} \times \mathbf{r}_{\tau}\right|^{-1} \mathbf{f} \cdot\left(\mathbf{v}_{0}-R \boldsymbol{\omega}_{\tau} \times \mathbf{e}_{3}+\boldsymbol{\omega}_{v} \times \mathbf{r}_{\tau}\right),
$$

where the normal pressure accounting for the rolling effect is represented by the linear approximation [23]:

$$
\sigma_{v}=\sigma_{0}\left[1+\left(\mathbf{r}_{\tau} \times \mathbf{h} \cdot \boldsymbol{\omega}_{\tau} /\left|\boldsymbol{\omega}_{\tau}\right|\right) \cdot \mathbf{e}_{3}\right]
$$

Here $\sigma_{0}=\sigma_{v}\left(\boldsymbol{\omega}_{\tau}=0\right)$, and $\mathbf{h}=h_{\alpha \beta} \mathbf{e}^{\alpha} \mathbf{e}^{\beta}$ is the "rolling friction tensor" for the anisotropic elastic body; we assume it being homogeneous and positively defined:

$$
\mathbf{h} \neq \mathbf{h}(M) ; \quad \forall \boldsymbol{\omega}_{\tau}=\boldsymbol{\omega}_{\tau}(\mathbf{q}) \quad \boldsymbol{\omega}_{\tau}^{\mathrm{T}} \cdot \mathbf{h} \cdot \boldsymbol{\omega}_{\tau}>0 .
$$

The static normal contact stress $\sigma_{0}$ is determined by the solution of the appropriate contact problem of elasticity theory in quasi-static statement. Thus, the model (5)-(8) allows one to use the static solution as a first approximation to model the coupled rolling and sliding friction, and the complex modeling of the dynamics of contact interaction is not required.

Now, accounting the rolling effect on the contact pressure on the basis of the formula (6) and using the formula (5) for the tangential contact stress that accounts by-turn both sliding (this term is denoted as $\tau_{1}$ ) and spinning (this one is denoted as $\tau_{2}$ ), we obtain finally the local model of the anisotropic dry friction in case of the combined kinematics:

$$
\begin{gathered}
\boldsymbol{\tau}=\boldsymbol{\tau}_{1}+\boldsymbol{\tau}_{2} ; \\
\boldsymbol{\tau}_{1}=-\left|\sigma_{0}\right|\left[1+\left(\mathbf{r}_{\tau} \times \frac{\mathbf{h} \cdot \boldsymbol{\omega}_{\tau}}{\left|\boldsymbol{\omega}_{\tau}\right|}\right) \cdot \mathbf{e}_{3}\right] \frac{\mathbf{f} \cdot\left(\mathbf{v}_{0}-R \boldsymbol{\omega}_{\tau} \times \mathbf{e}_{3}\right)}{\left|\mathbf{v}_{0}-R \boldsymbol{\omega}_{\tau} \times \mathbf{e}_{3}+\boldsymbol{\omega}_{v} \times \mathbf{r}_{\tau}\right|} \\
\boldsymbol{\tau}_{2}=-\left|\sigma_{0}\right|\left[1+\left(\mathbf{r}_{\tau} \times \frac{\mathbf{h} \cdot \boldsymbol{\omega}_{\tau}}{\left|\boldsymbol{\omega}_{\tau}\right|}\right) \cdot \mathbf{e}_{3}\right] \frac{\mathbf{f} \cdot\left(\boldsymbol{\omega}_{\tau} \times \mathbf{r}_{\tau}\right)}{\left|\mathbf{v}_{0}-R \boldsymbol{\omega}_{\tau} \times \mathbf{e}_{3}+\boldsymbol{\omega}_{v} \times \mathbf{r}_{\tau}\right|}
\end{gathered}
$$




\subsection{The global model of the anisotropic dry friction under combined kinematics}

The dynamic interaction of the slightly deformed rigid body with the rough support plane is defined by the normal reaction $\mathbf{N}$, the resultant vector of tangent forces $\mathbf{T}$, the anti-rolling couple $\mathbf{M}_{\tau}$, and the dry friction torque $\mathbf{M}_{v}$. These quantities are obtained by integration of the normal contact stress (6) as well as the summary tangential stress (8) over the contact area $S$ :

$$
\mathbf{N}=\int_{S} \sigma_{v} \mathbf{e}_{3} d S, \quad \mathbf{M}_{\tau}=\int_{S} \sigma_{v} \mathbf{r}_{\tau} \times \mathbf{e}_{3} d S, \quad \mathbf{T}=-\int_{S} \boldsymbol{\tau} d S, \quad \mathbf{M}_{v}=-\int_{S} \mathbf{r}_{\tau} \times \boldsymbol{\tau} d S
$$

In the formula (9), the resultant force $\mathbf{N}_{0}$ of the static contact pressure $\sigma_{0}$ and it's variation $\mathbf{N}_{1}$ induced by the rolling effect can be written as follows:

$$
\mathbf{N}_{0}=N_{0} \mathbf{e}_{3}, N_{0}=\int_{S} \sigma_{0} d S, \mathbf{N}_{1}=\int_{S} \sigma_{0} \frac{\mathbf{r}_{\tau} \times\left(\mathbf{h} \cdot \boldsymbol{\omega}_{\tau}\right)}{\left|\boldsymbol{\omega}_{\tau}\right|} d S=N_{1} \mathbf{e}_{3}, N_{1}=-\mathbf{S}_{\sigma} \cdot \mathbf{h} \cdot \frac{\boldsymbol{\omega}_{\tau}}{\left|\boldsymbol{\omega}_{\tau}\right|}
$$

We have the similar formulae for the anti-rolling couple where the "static" anti-rolling couple (that vanishes not in case of the rolling asymmetry of the body) and the "dynamic" one are defined by the formulae:

$$
\mathbf{M}_{\tau}^{0}=\int_{S} \sigma_{0} \mathbf{r}_{\tau} \times \mathbf{e}_{3} d S=\mathbf{S}_{\sigma} ; \quad \mathbf{M}_{\tau}^{1}=\int_{S} \sigma_{0} \mathbf{r}_{\tau} \times \frac{\mathbf{r}_{\tau} \times\left(\mathbf{h} \cdot \boldsymbol{\omega}_{\tau}\right)}{\left|\boldsymbol{\omega}_{\tau}\right|} d S=-\mathbf{J}_{\sigma} \cdot \mathbf{h} \cdot \frac{\boldsymbol{\omega}_{\tau}}{\left|\boldsymbol{\omega}_{\tau}\right|}
$$

Here the first moment vector $\mathbf{S}_{\sigma}$ and the inertia moment tensor $\mathbf{J}_{\sigma}$ of the plane area of contact $S$ with the static contact pressure distribution $\sigma_{0}$ are introduced:

$$
\mathbf{S}_{\sigma}=\int_{S} \sigma_{0} \epsilon_{\alpha \beta} \xi^{\beta} \mathbf{e}^{\alpha} d S, \mathbf{J}_{\sigma}=\int_{S} \epsilon_{\alpha \beta} \xi^{\beta} \epsilon_{\gamma \delta} \xi^{\delta} \mathbf{e}^{\alpha} \otimes \mathbf{e}^{\beta} d S, \epsilon_{\alpha \beta}=\left(\mathbf{r}_{\alpha} \times \mathbf{r}_{\beta}\right) \cdot \mathbf{e}_{3}, \alpha, \beta, \gamma, \delta=1,2 .
$$

The homogeneity of the tensor (7) and the formulae (9)-(11) lead to the vanishing as well normal reaction $\mathbf{N}_{1}$ as the rolling initiation moment $\mathbf{M}_{\tau}^{0}$ in the frame $O \xi^{1} \xi^{2}$ attached to the center of the figure $S$, therefore we have the following formulae for the normal reaction and anti-rolling couple: $\mathbf{N}=N_{0} \mathbf{e}_{3} ; \quad \mathbf{M}_{\tau}=-\mathbf{J}_{\sigma} \cdot \mathbf{h} \cdot\left(\boldsymbol{\omega}_{\tau} /\left|\boldsymbol{\omega}_{\tau}\right|\right)$.

The resultant vector of the anisotropic dry friction under combined kinematics can be now expressed through the following terms:

$$
\begin{gathered}
\mathbf{T}=\sum_{k=1}^{4} \mathbf{T}_{k}, \mathbf{T}_{1}=-\int_{S} \sigma_{0} \frac{\mathbf{f} \cdot \mathbf{v}_{S}}{\left|\mathbf{v}_{S}+\boldsymbol{\omega}_{v} \times \mathbf{r}_{\tau}\right|} d S, \mathbf{T}_{2}=-\int_{S} \sigma_{0}\left[\frac{\mathbf{r}_{\tau} \times\left(\mathbf{h} \cdot \boldsymbol{\omega}_{\tau}\right)}{\left|\boldsymbol{\omega}_{\tau}\right|} \cdot \mathbf{e}_{3}\right] \frac{\mathbf{f} \cdot \mathbf{v}_{S}}{\left|\mathbf{v}_{S}+\boldsymbol{\omega}_{v} \times \mathbf{r}_{\tau}\right|} d S \\
\mathbf{T}_{3}=-\int_{S} \sigma_{0} \frac{\mathbf{f} \cdot\left(\boldsymbol{\omega}_{v} \times \mathbf{r}_{\tau}\right)}{\left|\mathbf{v}_{S}+\boldsymbol{\omega}_{v} \times \mathbf{r}_{\tau}\right|} d S, \mathbf{T}_{4}=-\int_{S} \sigma_{0}\left[\frac{\mathbf{r}_{\tau} \times\left(\mathbf{h} \cdot \boldsymbol{\omega}_{\tau}\right)}{\left|\boldsymbol{\omega}_{\tau}\right|} \cdot \mathbf{e}_{3}\right] \frac{\mathbf{f} \cdot\left(\boldsymbol{\omega}_{v} \times \mathbf{r}_{\tau}\right)}{\left|\mathbf{v}_{S}+\boldsymbol{\omega}_{v} \times \mathbf{r}_{\tau}\right|} d S,
\end{gathered}
$$

Here $\mathbf{T}_{1}$ is the static dry friction force, $\mathbf{T}_{2}$ is the supplementary quasi-static dry friction force resulted by the rolling effect, $\mathbf{T}_{3}$ is the supplementary dry friction force due to the spin and the term $\mathbf{T}_{4}$ denotes the variation of the dry friction force due to the coupling of the rolling and spinning of the body.

The torque of the anisotropic dry friction under combined kinematics is also represented as a sum of four terms: 


$$
\begin{gathered}
\mathbf{M}_{v}=\sum_{k=1}^{4} \mathbf{M}_{k}, \mathbf{M}_{1}=-\int_{S} \sigma_{0} \frac{\mathbf{r}_{\tau} \times\left(\mathbf{f} \cdot \mathbf{v}_{S}\right)}{\left|\mathbf{v}_{S}+\boldsymbol{\omega}_{v} \times \mathbf{r}_{\tau}\right|} d S, \mathbf{M}_{2}=-\int_{S} \sigma_{0} \frac{\mathbf{r}_{\tau} \times\left(\mathbf{f} \cdot \mathbf{v}_{S}\right)}{\left|\mathbf{v}_{S}+\boldsymbol{\omega}_{v} \times \mathbf{r}_{\tau}\right|} d S, \\
\mathbf{M}_{3}=-\int_{S} \sigma_{0} \frac{\mathbf{r}_{\tau} \times\left[\mathbf{f} \cdot\left(\boldsymbol{\omega}_{v} \times \mathbf{r}_{\tau}\right)\right]}{\left|\mathbf{v}_{S}+\boldsymbol{\omega}_{v} \times \mathbf{r}_{\tau}\right|} d S, \mathbf{M}_{4}=-\int_{S} \sigma_{0}\left[\frac{\mathbf{r}_{\tau} \times\left(\mathbf{h} \cdot \boldsymbol{\omega}_{\tau}\right)}{\left|\boldsymbol{\omega}_{\tau}\right|} \cdot \mathbf{e}_{3}\right] \frac{\mathbf{r}_{\tau} \times\left[\mathbf{f} \cdot\left(\boldsymbol{\omega}_{v} \times \mathbf{r}_{\tau}\right)\right]}{\left|\mathbf{v}_{S}+\boldsymbol{\omega}_{v} \times \mathbf{r}_{\tau}\right|} d S
\end{gathered}
$$

Here $\mathbf{M}_{1}$ is the dry friction torque under the pure sliding that is resulted by the coupling of the slip and spin, the formula $\mathbf{M}_{2}$ defines the supplementary quasi-static dry friction torque resulted by the rolling effect on the slip, the "proper static" dry friction torque is defined by the formula $\mathbf{M}_{3}$ and it's variation due to the coupling of the rolling and spinning is given by the term $\mathbf{M}_{4}$. The vector $\mathbf{v}_{S}$ denotes the summary velocity in the point $M \in S$ :

$$
\mathbf{v}_{S}=\mathbf{v}_{0}-R \boldsymbol{\omega}_{\tau} \times \mathbf{e}_{3} ; \quad\left|\mathbf{v}_{\Sigma}\right|^{2}=v_{S}^{2}+2 \mathbf{v}_{S} \cdot\left(\boldsymbol{\omega}_{v} \times \mathbf{r}_{\tau}\right)+\left(\boldsymbol{\omega}_{v} \times \mathbf{r}_{\tau}\right) \cdot\left(\boldsymbol{\omega}_{v} \times \mathbf{r}_{\tau}\right) .
$$

Thus, the invariant formulation for the coupled dry friction theory is obtained. The constructed model describes the coupling effects under combined sliding, spinning, and rolling of the deformed rigid body with the finite area of contact with the support plane. The invariant relationships (9) can be rewritten in the coordinate form by the appropriate choice of the main frame.

\section{Approximate model of the rolling wheel}

Let us consider the orthotropic dry friction given by the tensor $\mathbf{f}=f\left(\begin{array}{ll}1 & 0 \\ 0 & \kappa\end{array}\right), f \neq 0, \kappa \neq 0$.

Here $f$ and $\kappa f$ are the principal components of the tensor $\mathbf{f}$. Let us introduce the frame $O x^{1} x^{2}$ attached to the centroid of the contact spot $S$; the corresponding base vectors $\mathbf{e}_{1}$ and $\mathbf{e}_{2}$ are collinear to the principal directions of the tensor $\mathbf{f}$. The static contact pressure symmetry, $\sigma_{0}\left(x^{1}, x^{2}\right)=\sigma_{0}\left( \pm x^{1}, \pm x^{2}\right)$, as well as the rolling friction isotropy are assumed.

Let us consider the motion defined by the longitudinal velocity $\mathbf{v}_{0}=V_{0} \mathbf{e}_{1}^{\prime}$ along the axis $O X^{1}$ of the global rest frame (for more details, see [16]), the rolling angular velocity $\boldsymbol{\omega}_{\tau}=-\omega_{\tau} \mathbf{e}_{2}$, and the spinning velocity $\omega_{v}$. Such a situation corresponds to the rolling of the wheel with the thread characterized by the friction factors $t$ along the tread and $\kappa f$ across it. Let us also consider here only circular area of contact with the radius $R$.

The quantities (13)-(14) can now be simplified as it was implemented in $[10,11,16$, 17]. The resultant force vector can be represented as $\mathbf{T}=T_{\|} \mathbf{e}_{1}+T_{\perp} \mathbf{e}_{2}$, so that $T_{\|}$is the longitudinal and $T_{\perp}$ is the lateral friction force. It was shown [7-11, 16] that the last one is due to the coupling effects. As a result, we have the following formulae:

$$
T_{\|}=F_{0} \frac{v_{s}}{\sqrt{v_{s}^{2}+a u^{2}}}, T_{\perp}=h \kappa F_{0} \frac{u v_{s}}{\sqrt{\left(u^{2}+b v_{s}^{2}\right)\left(v_{s}^{2}+a u^{2}\right)}}, M_{v}=M_{0} \frac{u}{\sqrt{u^{2}+m v_{s}^{2}}}
$$

Here $u=\omega_{v} R, F_{0}=f N_{0}$ is the longitudinal resting friction force, the resting friction torque can be computed as follows: $M_{0}=\pi(1+\kappa) f \int_{0}^{1} \sigma_{0}(r) r^{2} d r$. Here the dimensionless 
radial coordinate is introduced. For the factors $a, b, m$ we have following formulae analogous to these published in [17].

\section{Accounting for the contact pressure distribution}

The contact pressure distribution close to the real one as well as the contact spot diameter can be obtained on the basis of the finite element model of a tire; several levels of accuracy of the model can be considered. The first and simplest one consists in the numerical simulation of the quasi-static nonlinear deforming of a tire using the plane elasticity problem statement for the tire cross-section [15]. The contact pressure distribution computed for several levels of the vertical load or the radial deformation of the tire are then interpolated analytically.

The polynomial interpolation of the finite element solution $\sigma_{0 i}$ is constructed as follows: $\sigma_{0}(r) \approx \sigma_{0}^{k} p_{k}(r), \quad r \in[-1,1], \quad k=0,2 \ldots N \in \mathbb{N}$, where $r$ is the dimensionless coordinate in the contact spot and $p_{k}(r)$ are Legendre polynomials. The contact pressure distribution is assumed to be symmetric, $\sigma_{0}(r)=\sigma_{0}(-r)$. The factors $\sigma_{0}^{k} \in \mathbb{R}$ are obtained from the solution of the quadratic programming problem: $G_{k m} \sigma_{0}^{k} \sigma_{0}^{m} \rightarrow \min , G_{k m}=2 \delta_{k m} /(2 k+1)$ with the following restrictions: $\sigma_{0}^{k} p_{k}\left(r_{i}\right) \leq \sigma_{0 i}$, $\sigma_{0}^{k} p_{k}(1)=0, \sigma_{0}^{k} \int_{-1}^{1} p_{k}(r) d r=\sum_{i=2}^{n}\left(\sigma_{0 i}+\sigma_{0 i-1}\right)\left(r_{i}+r_{i-1}\right)$, where the first one corresponds to the lower approximation of the FEM solution, the second one corresponds to the condition $\left.\sigma_{0}\right|_{\partial S}=0$, and the third one guarantees the equivalence of the normal reaction following from the interpolation and the load applied to the FE model. The typical contact pressure distributions and its approximations are presented in [17].

The high level of vertical loads results the O-shaped area of contact where the contact pressure vanishes near the center of a spot [15]. In such a situation, the higher-order approximation may be required. On the other hand, the strongly deformed state of a tire leads to the accounting for the tangential deformation and the elastic forces in the pneumatics, therefore this model become combined with the Keldysh concept.

These investigations were carried out within the framework of the state program of the scientific research works on the section No.: AAAA-A17-117021310382-5 and were supported by the Moscow Institute of Physics and Technology (State University).

\section{References}

[1] M.V. Keldysh, Selected Works, Mechanics, Nauka, Moscow (1985)

[2] A.A. Zagordan, Journal "Trudy MAI". Moscow Aviation Institute 47:11 pp (2011) (in Russian)

[3] A.A. Zagordan, S.I. Zhavoronok, Nelineiny Mir 10, 646-656 (2011) (in Russian)

[4] P. Contensou, Kreisel probleme Gyrodynamics: IUTAM Symp. Celerina, 1962, Springer, Berlin, 201-216 (1963)

[5] Th. Erissmann, ZAMP 5, 355-388 (1954) (in German)

[6] V.Ph., Zhuravlev, D.M. Klimov, Mech. Solids 45(3), 324-330 (2010)

[7] V.V. Andronov, V.Ph. Zhuravlev, Dry Friction in Problems of Mechanics, NITs Reg. Khaot. Dinam., Moscow-Izhevsk (2010) (in Russian)

[8] V.Ph. Zhuravlev, J. Appl. Math. Mech. 62(5), 762-767 (1998) 
[9] V.Ph. Zhuravlev, A.A. Kireenkov, Mech. Solids 40(2), 1-10 (2005)

[10]A.A. Kireenkov, Doklady Akademii Nauk 419(6), 759-762 (2008)

[11] A.A. Kireenkov, Doklady Akademii Nauk 441(6), 750-755 (2011)

[12] N.S. Bernikova, A.A. Zagordan, S.I. Zhavoronok, Proc. of the 8th European Solid Mechanics Conf. (ESMC-2012), R.W. Ogden, G.A. Holzapfel (Eds.), Graz, Austria (2012)

[13]N.S. Bernikova, A.A. Zagordan, S.I. Zhavoronok, Proc. 8th European Nonlinear Dynamics Conf. (ENOC-2014), Vienna, Austria (2014)

[14] N.S. Bernikova, E.V. Stepanov, A.A. Zagordan, S.I. Zhavoronok, Proc. 11th Int. Conf. on Vibration Probl. (ICOVP-2013), Z. Dimitrovova, J. de Almeida, R. Gonçalves (Eds.), AMPTAC, Lisbon, Portugal (2013), p. 290.

[15] S.E. Bogoslovskii, N.N Kurdyumov, Proceedings of the TSU. Technical Sciences 8(2), 138-147 (2015)

[16]A.A. Kireenkov, Proc. 5th European Nonlinear Dynamics Conf. ENOC-2005, Eindhoven, Netherlands (2005).

[17]A.A. Kireenkov, S.I. Zhavoronok, Int. J. Mech. Sci. DOI 10.1016/j.ijmecsci. 2017.02.004 (2017)

[18] V.V. Kozlov, Nonlinear Dynamics 6(4), 855-868 (2010) (in Russian)

[19] V.G. Vil'ke, Mech. Solids 72, 3-8 (2008)

[20]A. Zmitrowiecz, Int. J. Sol. Struct. 25(8), 837-862 (1989)

[21]N. Dmitriev, O. Silantyeva, Vestnik St. Petersburg University. Mathematics 46(1), 9298 (2016)

[22] O. Silantyeva, N. Dmitriev, CRC Press 511-515, (2016)

[23]A.A. Kireenkov, COUPLED PROBLEMS 2015-Proceedings of the 6th International Conference on Coupled Problems in Science and Engineering, Venice, Italy, 203-209 (2015) 